\title{
SINGLE-PARTICLE INDUCTIVELY COUPLED PLASMA MASS SPECTROSCOPY ANALYSIS OF SIZE AND NUMBER CONCENTRATION IN MIXTURES OF MONOMETALLIC AND BIMETALLIC (CORE-SHELL) NANOPARTICLES
}

Ruth C. Merrifield ${ }^{\dagger}$, Chady Stephan ${ }^{\ddagger}$ and Jamie R. Lead ${ }^{\dagger}$

† Center for Environmental Nanoscience and Risk (CENR) Arnold School of Public Health University of South Carolina, USA

‡ PerkinElmer Canada, 501 Rowntree Dairy Rd, Unit 6, Woodbridge, ON L4L 8H1, Canada 


\section{ABSTRACT}

It is challenging to separate and measure the physical and chemical properties of monometallic and bimetallic engineered nanoparticles (NPs), especially when mixtures are similar in size and at low concentration. We report that single particle inductively coupled mass spectroscopy (SP. ICP-MS), alongside field flow fractionation (FFF), has allowed for the accurate measurement of size and particle number concentrations of mixed metallic nanoparticles (NPs) containing monometallic NPs of gold $(\mathrm{Au})$ and silver $(\mathrm{Ag})$ and a bimetallic core-shell structured NP (Au@Ag) of equivalent size. Two sets of these NPs were measured. The first contained only 60 nm particles, where the Au@Ag NP had a 30 nm core and $15 \mathrm{~nm}$ shell to make a total diameter of $60 \mathrm{~nm}$. The second contained only $80 \mathrm{~nm}$ particles (Au@Ag NP core particle of $50 \mathrm{~nm}$ with a $15 \mathrm{~nm}$ shell). FFF separation was used here as a sizing technique rather than a separation technique. It was used to confirm that suspensions containing either individual or mixtures of the Au $60 \mathrm{~nm}$, Ag $60 \mathrm{~nm}$ and AuAg $60 \mathrm{~nm}$ suspensions eluted together and were of the same size. Similarly, FFF was used to show that suspensions containing individual or mixtures of the equivalent $80 \mathrm{~nm}$, eluted together and were of the same size. Although the $60 \mathrm{~nm}$ and $80 \mathrm{~nm}$ suspensions did not elute at the same time they were not run together. SP-ICP-MS is then used to identify the size and concentration of the particles within the suspension. Successful separation of the NPs was effected and the limits of the instrument were obtained. 


\section{Introduction}

Nanoparticles (NP) are widely used in an array of applications ${ }^{1,2}$ due to their unique physicalchemical properties. These properties can be tuned by changing the size, shape and composition of the NP ${ }^{3,4}$. It has always been a challenge to fully characterize suspensions of monometallic NPs, where a multimethod approach will yield the most reliable results ${ }^{5,6}$. This process becomes increasingly difficult for more complex bimetallic NPs, or mixtures thereof, used in some applications or for those that require a mixture of different NPs. There are a variety of techniques that have been used for the determination of particle diameter, composition and particle number concentration (part. $\mathrm{mL}^{-1}$ ) of engineered NPs; including dynamic light scattering (DLS), field flow fractionation $(F F F)^{7,8}$, ultraviolet-visible spectroscopy $(\mathrm{UV}-\mathrm{Vis})^{9}$, multi-angle light scattering (MALS) $)^{10}$ and transmission electron microscopy (TEM) ${ }^{11}$. More recently single-particle inductively coupled mass spectroscopy (SP-ICP-MS) ${ }^{12}{ }^{13}$ has been applied although it has not been fully developed in this area. All these techniques have their own strengths and weaknesses as well as measuring size and/or composition in different ways. One of the most difficult properties to routinely measure in a NP suspension is particle number concentration. $^{12,} 14$ This property can be measured Nanosight ${ }^{15}$, although its accuracy is questionable ${ }^{16,17}$. In fact all current methods are arduous and inaccurate with limitations in measurement; for instance, bimetallic (core-shell) NPs cannot be measured. DLS can be used to quantify size for some systems, as can FFF, which must be used with a detector, such as UV-Vis. Coupling FFF to an ICP-MS provides a lower detection limit compared with other methods ${ }^{18}$. However all these detection techniques are unable to discriminate mixtures of monometallic and/or bimetallic particles. 
SP-ICP-MS is an excellent technique for the detection of metallic NPs providing data on size, size distribution, particle number concentration and other parameters ${ }^{13}$ and giving such data at low concentrations, which are important for many applications such as environmental measurements $^{19,20}$. Advances in the measurement within shorter dwell times have led to improvements in detection by SP-ICP-MS and have also enabled for the qualitative measurement of two elements within the same NP ${ }^{21}$. FFF separates NPs by their hydrodynamic diameters. However FFF is unable to distinguish between mixtures of NPs with the same diameter but different compositions. Here we use FFF to establish whether mixtures of particles are nominally of the same size. This enables us to conclude that if we measure two elements with SP-ICP-MS together in that fraction that are smaller than the FFF size, and the particle number concentrations of both metals are equal then they will be from a bimetallic particles. If the sizes of the 2 elements are comparable to the FFF size and the particle number concentrations are different then the suspension will contain a mixture of monometallic NPs. We are able to show that a sizing and particle number counting method using SP-ICP-MS can be used to measure more complex suspensions of NPs within these fractions, and can discriminate between mixtures of monometallic Au and Ag NPs and bimetallic Au@Ag (core shell) NPs, yielding particle sizes and particle numbers concentrations for each constituent. The limitations to such resolution are described. 


\section{Experimental}

\section{Nanoparticles (NPs)}

NP suspensions for this study were purchased from Nanocomposix (San Diego, CA, USA) and the National Institute of Standards Technology (NIST, Gaithersburg MD, USA). Particles with several sizes and compositions were used including 60 nm Ag, 60 nm Au, 60 nm Au@Ag (core size 30 nm, shell thickness 15 nm), 80 nm Ag, 80 nm Aug and 80 nm Au@Ag (core size 50 nm, shell thickness $15 \mathrm{~nm}$ ), purchased from Nanocomposix, were used either as single particle suspensions or mixtures. In addition, Au $30 \mathrm{~nm}$ and Au $60 \mathrm{~nm}$ NPs from NIST were used as certified reference materials. NP concentrations and other parameters were provided by the supplier (See supporting information S1 Table 1) and were diluted to between 10,000 and 100,000 part. $\mathrm{mL}^{-1}$ for SP-ICP-MS analysis, based on manufacturers data. For FFF analysis, NPs were used at concentrations of either the nominal concentrations of the stocks (See supporting information S1 Table 1) or diluted to 1,000,000 part. $\mathrm{mL}^{-1}$, depending on detector and injection volumes used. It was found that the given particle concentrations and sizes were not always as the manufacturer stated and in some cases the particle numbers were higher or lower than what was expected.

\section{Field flow fractionation (FFF)}

An eclipse DualTech hollow fiber flow field-flow fractionation separation system (Wyatt technology, Santa Barbara, CA, USA) FFF was used in combination with a Agilent 1260 Infinity LC pump and auto sampler introduction system and an online UV detector (Agilent 1260 infinity, Agilent technologies, Santa Clara, Ca, USA). 
A $10 \mathrm{KDa}$ PES hollow fiber was used as a separation channel with a diameter of $0.8 \mathrm{~mm}$ and length of $17 \mathrm{~cm}$ (purchased from Wyatt). The detector flow was $0.5 \mathrm{~mL} \mathrm{~min}^{-1}$, focus flow of 0.2

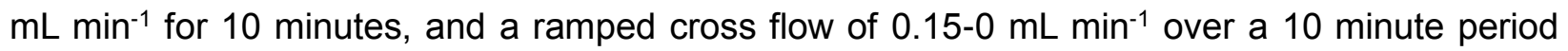
the particles eluted between 0.1 and $0.6 \mathrm{~mL} \mathrm{~min}^{-1}$ cross flow) and injection volumes of 2-20 $\mu \mathrm{L}$. The carrier solution was a sodium citrate solution at a concentration of $0.2 \mathrm{mM}$.

\section{Inductively coupled plasma mass spectroscopy (ICP-MS)}

A PerkinElmer NexION 350D ICP-MS was used with a Meinhard nebulizer, cyclonic (standard) spray chamber, a $2 \mathrm{~mm}$ ID injector and a glass torch. A RF power of $1600 \mathrm{~W}$, auxiliary gas flow of $1.2 \mathrm{~mL} \mathrm{~min} \mathrm{~m}^{-1}$ and nebulizer gas flow of $1.12 \mathrm{~mL} \mathrm{~min}-1$ was used for measurements. The sample uptake was $0.49 \mathrm{~mL} \mathrm{~min}^{-1}$. For single particle measurements (SP-ICP-MS) a dwell time of $50 \mu$ s was used with 30 to 60 s second sampling times.

\section{Standards}

1, 2, 3 and $4 \mathrm{ppb}$ ionic Au and Ag standards were used as ionic calibrations. NIST Au standards of $30 \mathrm{~nm}$ and $60 \mathrm{~nm}$ were used for Au particle calibrations at concentrations of 100,000 part. $\mathrm{mL}^{-1}$. The NIST $60 \mathrm{~nm}$ Au particles were used to determine transport efficiency. 


\section{Results and Discussion}

NIST standards of nominally 30 and $60 \mathrm{~nm}$ certified reference materials were used to quantify the accuracy of the SP-ICP-MS system (Data in Figure S2). The diameter and size distribution measured by TEM and quoted by NIST for the nominally 30 and $60 \mathrm{~nm}$ standards are slightly smaller than the nominal value: $27.6( \pm 2.1)$ and $56.3( \pm 1.5) \mathrm{nm}$ respectively. Diameters of the same NPs measured in this study were $26.9( \pm 0.3) \mathrm{nm}$ and $57.3( \pm 0.1)$, in excellent agreement with the stated values from NIST. The $60 \mathrm{~nm}$ CRM were measured over the course of the experiment, and the particle number concentrations for these suspensions were consistently within $1 \%$ of those originally measured for the TE. This along with the consistency of the size showed gave confidence in the stability of the machine throughout the experiment. Additionally, the particle number concentrations of the $30 \mathrm{~nm}$ certified reference materials were within $3 \%$ of the values stated by NIST, showing that the particle number counting and particle sizing by SPICP-MS was in good agreement with well characterized NPs.

Six test NP suspensions purchased from commercial sources (Ag, Au and Au@Ag; all at 60 and 80 nominal sizes) were analyzed by SP-ICP-MS to derive the particle size and concentration of each individual suspension for later reference. The size histograms of the NIST samples (Figure S2 A and B) very monodisperse samples, while the Nanocomposix NPs have a higher degree of polydispersity, sometimes showing bimodal distributions (examples shown in Figures S3, S4 and S5). For instance, peaks at $60.9( \pm 0.1) \mathrm{nm}$ and $47.5( \pm 0.1) \mathrm{nm}$ for the nominally Au-60 $\mathrm{nm}$ NPs (S3-B), $73.5( \pm 0.1)$ nm and $59.6( \pm 0.4)$ nm for the Ag signal of the Au@Ag-80 nm particles $(\mathrm{S} 4-\mathrm{C})$ and $78.1( \pm 0.1) \mathrm{nm}$ and $60.4( \pm 0.1) \mathrm{nm}$ for the nominally Au-80 nm NPs (S4-B) were all observed by SP-ICP-MS. In all cases, the larger peak is closest to the manufacturer supplied data (Table S5-1). It is also evident that the secondary peak contains a substantial number of the particles: $65.8 \%, 65.6 \%$ and $54.2 \%$ of the particles are related to the larger diameter NP 
peak in the above examples. To simplify data analysis, we fitted a Gaussian curve to the size distributions and used these fits for data interpretation (seen in Figures S3 and S4).

Figure $1 \mathrm{~A}$ and $\mathrm{B}$ show the measured particle diameters (Figure $1 \mathrm{~A}$ ) and measured particle number concentrations (Figure $1 \mathrm{~B}$ ) for the individual NP suspensions. The total particle diameters for the separate Ag or Au NPs are $73.5(3.9) \mathrm{nm}, 76.2(0.2) \mathrm{nm}, 54.7$ (0.2) and 55.5 (0.3) $\mathrm{nm}$ for the nominally Au-80 nm, Ag-80 nm, Au-60 nm and Ag-60 nm particles respectively. The actual stated values from the manufacturers are 81.2 (10.5), 78.9 (10.5), 60.6 (5.9) and 59.6 (5.8), respectively. In general, the data is different, but not significantly so, largely due to the poor precision in the manufacturer's data.

SP-ICP-MS measures total number of ions generated from individual NP and does not account for geometry of the NP. Thus for bimetallic (core-shell) particles, two sizes are measured that assume a solid Au and a solid Ag NP (Discussion in supporting information S6). Accounting for the geometry (calculations can be found in Merrifield and Lead 2016 22 ), the number of ions for the Au and Ag solid NPs translate into a larger core-shell structured NP of $57.8( \pm 0.3)$ and 74.3 ( \pm 0.5$)$ for the Au@Ag-60 nm and Au@Ag-80 nm respectively. These calculated sizes can be directly compared to the manufactures (TEM) measurements of $60.8( \pm 6.3)$ and $78.2( \pm 8.8)$, showing good agreement. In this case we knew the composition of the NP to have an Au core and $\mathrm{Ag}$ shell, however calculations can be made for the total size if the reverse were true (an Ag core and Au shell), or for an alloy NP of the same composition and minimal exploration with another technique such as STEM/EDS would be required to verify this.

Figure 1B shows the particle number concentrations for each of the particle suspensions. All of the suspensions were diluted such that their concentration was expected to be 100,000 particles 
$\mathrm{mL}^{-1}$ and then back calculate to the original concentrations. It is evident that the manufacturer's data for most suspensions is in agreement with our data, although data for the Au-80 and Ag-60 were not in agreement with the SP-ICP-MS and we corrected for this in subsequent analyses (Table S1). For the core-shell NPs, the number concentrations of both $\mathrm{Ag}$ and $\mathrm{Au}$ signals were identical (within $0.5 \%$ of each other), suggesting that the signals either come from the same bimetallic particle or equal amounts of separate monometallic particles, although the latter is unlikely. FFF data on the individual bimetallic suspensions showed only a single elution peak, indicating that both elements elute at the same time. Figure S7 shows the $80 \mathrm{~nm}$ AuAg NP suspension eluting in a tight peak less than a minute after the $80 \mathrm{~nm}$ standard polystyrene beads. There is also a clear separation between the $80 \mathrm{~nm}$ and $40 \mathrm{~nm}$ PS standards meaning that if the manufactures had not coated the Au cores correctly in Ag we would expect to see a signal eluting just after the $40 \mathrm{~nm}$ PS standard. Thus, confirming that the sample contains only a bimetallic (Au@Ag) NPs and not mixtures of Ag and Au NPs.

Mixtures of Au@Ag, Au and Ag NPs in SP-ICP-MS provide an extremely challenging test of the selectivity of the SP-ICP-MS. Typical SP-ICP-MS data for mixtures of the $80 \mathrm{~nm}$ particles and $60 \mathrm{~nm}$ particles are shown in Figure 2. The Au and Ag signals for the $80 \mathrm{~nm}$ mixture shown in Figure $2 \mathrm{~A}$ and $2 \mathrm{~B}$ respectively and the $\mathrm{Au}$ and $\mathrm{Ag}$ signals for the $60 \mathrm{~nm}$ mixtures are shown in Figure 2C and D respectively. The Gaussian fits for the single NP suspensions (taken from Figure S3 and S4) are shown. The dotted black lines represent the Gaussian fit for the monometallic NPs while the dashed lines show the bimetallic NPs. Figure $1 \mathrm{~A}$ and $\mathrm{C}$ show a good separation of the signal from the Au core particles at $46.0 \mathrm{~nm}$ (A dashed line) and $29.5 \mathrm{~nm}$ ( $C$ dashed line) and the Au monometallic particles at $76.2 \mathrm{~nm}$ (A dotted line) and $54.7 \mathrm{~nm}$ (C dotted line). This separation allows the size and concentration of the core particles (from the Au@Ag NPs) and the monometallic Au NPs to be quantified separately. 
Figures $2 \mathrm{~B}$ and $\mathrm{D}$ are the $\mathrm{Ag}$ signals for the $80 \mathrm{~nm}$ mixture and $60 \mathrm{~nm}$ mixture respectively. In contrast to the Au signals, the Ag shell signal (dashed lines) and Ag monometallic NP (dotted lines) overlap and they cannot be distinguished on the basis of SP-ICP-MS alone. In general, to successfully separate two Gaussian distributions a resolution of 1.2 or above needs to be achieved $^{23}$, below this the distributions are difficult to distinguish. This equates to the distance between peaks needing to be at least twice the average of the full width half maxima of each peak to accurately separate the two particle distributions and be able to measure size and particle number concentration. This is achieved for the Au signal in our mixtures (figure $2 \mathrm{~A}$ and C) but not in the Ag signals (Figure 2B and D).

Despite the lack of separation in the Ag signal it is still possible to calculate the particle number concentrations for all three NPs in the suspension using particle number counting. There is a large enough separation between the peaks in the Au signal to determine the particle number concentration for both the Au monometallic particle and the Au core from the bimetallic NP. The Ag data contains a component from both the Ag monometallic NP and Ag shell in the core-shell NPs. The particle number of the bimetallic NPs was calculated from the Au signal. By subtracting this signal this from the total Ag signal, the particle number concentration for the Ag monometallic NP can be calculated. By this process, it is possible to calculate the concentrations of all the NPs.

Subsequent to this initial analysis, five mixtures of these NPs suspensions were produced (see Methods for details). The NP diameters and number concentrations for the three mixtures containing Au 80 nm and Au@Ag 80 nm NPs are shown in Figure 3 A and B respectively. For these samples the Au signal contained information for both of the Au core and Au monometallic 
NPs, while the Ag signal only contained information from the Ag shell. The total measured NP number concentration was used as a check to ensure that the particles were not being over or under-represented. In all mixtures, the total number concentration measured for the Au signal is within $0.5 \%$ of that measured for the smaller and larger peak combined, showing that all particles are counted once. In all mixture ratios of the Au and Au@Ag NPs, the particle sizes and concentrations were fully resolved to their respective components, as shown in Figure 3. The \% of Au NPs that could be separated analytically from the Au@Ag NPs varied with Au:Au@Ag NP ratio. At 50:50 Au:Au@Ag 95\% of both NPs were analytically separated. At 80:20 Au:Au@Ag 100\% of the Au NPs were separated and only 84\% of the Au@Ag NPs, while the reverse is true for the 20:80 Au:Au@Ag mixture. In figure 3A the particle diameters for the Au-80 nm, Au-core and Ag shell were all measured within 3\% of those measured for the individual NPs. The particle number concentrations of the mixtures, after measurement and back-calculation from the mixtures were not significantly different from their expected values, based on Figures 1 and 2 and the mixture ratios used. Although mixtures in this case could be satisfactorily resolved, the challenge in back calculating to the true individual values from a mixture becomes more pronounced where the mixture ratios are high (or low) and where the NP samples are polydisperse. For these bimodal suspensions and at these mixture ratios, the actual and expected values are in good agreement, with $<20 \%$ difference in size and number concentration.

Figure 4 shows the measured sizes (Figure $4 \mathrm{~A}$ ) and calculated particle numbers (Figure $4 \mathrm{~B}$ ) for the $80 \mathrm{~nm}$ and $60 \mathrm{~nm}$ mixed NP suspensions i.e. a three component mixture of the $\mathrm{Ag}, \mathrm{Au}$ and Au@Ag NPs. The Au core and NP can be quantitatively resolved due to their differences in size. However, the Ag signal cannot be resolved as the Ag monometallic NPs and the Ag shell of the Au@Ag NPs are too similar. Neither the Au-monometallic NPs nor the total diameter for the 
Au@Ag NPs could be distinguished and calculated. However, the particle number concentrations for all three particles were calculated from the acquired data and are shown in figure 4B. The measured particle number concentrations for the $80 \mathrm{~nm}$ mixture are shown on the left hand side of the graphs in figure $4 \mathrm{~A}$ and $\mathrm{B}$ respectively. The particle numbers concentrations for the $80 \mathrm{~nm}$ mixture were approximately $66,100,53,400$ and 70,000 particles $\mathrm{mL}^{-1}$ for the Au, Ag and Au@Ag particles respectively. These particle number concentrations are within $5 \%$ of the original added amounts $\left(64,600,53,900\right.$ and 73,300 part. $\left.\mathrm{mL}^{-1}\right)$, showing excellent agreement. For the nominally $60 \mathrm{~nm}$ particles, concentrations were within $7 \%$ of the expected values $\left(107,000,65,800,37,800\right.$ part. $\mathrm{mL}^{-1}$ for the Au-60 nm, Ag-60 nm and Au@Ag$60 \mathrm{~nm}$ particles compared to the expected values of $100,000,66,000$ and 40,000 part. $\mathrm{mL}^{-1}$ ). The measured values for the particle sizes and the measured and corrected values for the particles number concentrations can be found in S8. 


\section{Conclusions}

In summary, we have shown by FFF that commercially brought monometallic (Au and $\mathrm{Ag}$ ) NPs and bimetallic (AuAg) NPs nominally of the same size elute together and have the same size distribution. We have also shown that FFF alone is unable to distinguish between either individual NPs or mixtures of these NPs. It was also shown that the SP-ICP-MS system is capable of collecting data on size and number concentration of complex mixtures of metal and core-shell metal NPs. The obtained data resolution shows that this method is an excellent method for NP metrology in complex systems, capable of adding substantial analytical capability to established multi-method approaches. ${ }^{17}$ 


\section{ACKNOWLEDGMENT}

The authors would like to acknowledge the National Science Foundation (1508931) along with PerkinElmer and the Center for Environmental Nanoscience and Risk, University of South Carolina, for financial support. 


\section{REFERENCES}

1. Stark, W. J.; Stoessel, P. R.; Wohlleben, W.; Hafner, A., Industrial applications of nanoparticles. Chemical Society Reviews 2015, 44, (16), 5793-5805.

2. Ghosh Chaudhuri, R.; Paria, S., Core/Shell Nanoparticles: Classes, Properties, Synthesis

Mechanisms, Characterization, and Applications. Chemical Reviews 2012, 112, (4), 2373-2433.

3. Lue, J.-T., A review of characterization and physical property studies of metallic nanoparticles. Journal of Physics and Chemistry of Solids 2001, 62, (9-10), 1599-1612.

4. Ju-Nam, Y.; Lead, J. R., Manufactured nanoparticles: An overview of their chemistry, interactions and potential environmental implications. Science of The Total Environment 2008, 400, (1-3), 396-414.

5. Baalousha, M.; Ju-Nam, Y.; Cole, P. A.; Gaiser, B.; Fernandes, T. F.; Hriljac, J. A.; Jepson, M. A.; Stone, V.; Tyler, C. R.; Lead, J. R., Characterization of cerium oxide nanoparticles-Part 1: Size measurements. Environmental Toxicology and Chemistry 2012, 31 , (5), 983-993.

6. Baalousha, M.; Ju-Nam, Y.; Cole, P. A.; Hriljac, J. A.; Jones, I. P.; Tyler, C. R.; Stone, V.; Fernandes, T. F.; Jepson, M. A.; Lead, J. R., Characterization of cerium oxide nanoparticlesPart 2: Nonsize measurements. Environmental Toxicology and Chemistry 2012, 31, (5), 9941003.

7. Ulrich, A.; Losert, S.; Bendixen, N.; Al-Kattan, A.; Hagendorfer, H.; Nowack, B.; Adlhart, C.; Ebert, J.; Lattuada, M.; Hungerbuhler, K., Critical aspects of sample handling for direct nanoparticle analysis and analytical challenges using asymmetric field flow fractionation in a multi-detector approach. Journal of Analytical Atomic Spectrometry 2012, 27, (7), 1120 1130.

8. Baalousha, M.; Stolpe, B.; Lead, J. R., Flow field-flow fractionation for the analysis and characterization of natural colloids and manufactured nanoparticles in environmental systems: A critical review. J. Chromatogr. A 2011, 1218, (27), 4078-4103.

9. Wilcoxon, J., Optical Absorption Properties of Dispersed Gold and Silver Alloy Nanoparticles. Journal of Physical Chemistry B 2009, 113, (9), 2647-2656.

10. Gigault, J.; Grass1, B.; Le Hécho, I.; Lespes, G., Accurate determination of the length of carbon nanotubes using multi-angle light scattering. Microchimica Acta 2011, 175, (3), 265-271.

11. Prasad, A.; Lead, J. R.; Baalousha, M., An electron microscopy based method for the detection and quantification of nanomaterial number concentration in environmentally relevant media. Science of the Total Environment 2015, 537, 479-486.

12. Shang, J.; Gao, X., Nanoparticle counting: towards accurate determination of the molar concentration. Chemical Society Reviews 2014, 43, (21), 7267-7278.

13. Laborda, F.; Jimenez-Lamana, J.; Bolea, E.; Castillo, J. R., Critical considerations for the determination of nanoparticle number concentrations, size and number size distributions by single particle ICP-MS. Journal of Analytical Atomic Spectrometry 2013, 28, (8), 1220-1232.

14. Li, M.; Guha, S.; Zangmeister, R.; Tarlov, M. J.; Zachariah, M. R., Method for Determining the Absolute Number Concentration of Nanoparticles from Electrospray Sources. Langmuir 2011, 27, (24), 14732-14739.

15. Malloy, A., Count, size and visualize nanoparticles. Materials Today 2011, 14, (4), 170173. 
16. Baalousha, M.; Sikder, M.; Prasad, A.; Lead, J.; Merrifield, R.; Chandler, G. T., The concentration-dependent behaviour of nanoparticles. Environmental Chemistry 2016, 13, (1), 13.

17. Domingos, R. F.; Baalousha, M. A.; Ju-Nam, Y.; Reid, M. M.; Tufenkji, N.; Lead, J. R.; Leppard, G. G.; Wilkinson, K. J., Characterizing Manufactured Nanoparticles in the Environment: Multimethod Determination of Particle Sizes. Environmental Science \& Technology 2009, 43, (19), 7277-7284.

18. Mitrano, D. M.; Barber, A.; Bednar, A.; Westerhoff, P.; Higgins, C. P.; Ranville, J. F., Silver nanoparticle characterization using single particle ICP-MS (SP-ICP-MS) and asymmetrical flow field flow fractionation ICP-MS (AF4-ICP-MS). Journal of Analytical Atomic Spectrometry 2012, 27, (7), 1131-1142.

19. Gottschalk, F.; Sonderer, T.; Scholz, R. W.; Nowack, B., Modeled Environmental Concentrations of Engineered Nanomaterials ( $\mathrm{TiO} 2, \mathrm{ZnO}, \mathrm{Ag}, \mathrm{CNT}$, Fullerenes) for Different Regions. Environmental Science \& Technology 2009, 43, (24), 9216-9222.

20. Fabrega, J.; Zhang, R.; Renshaw, J. C.; Liu, W.-T.; Lead, J. R., Impact of silver nanoparticles on natural marine biofilm bacteria. Chemosphere 2011, 85, (6), 961-966. 21. Montano, M. D.; Badiei, H. R.; Bazargan, S.; Ranville, J. F., Improvements in the detection and characterization of engineered nanoparticles using spICP-MS with microsecond dwell times. Environmental Science: Nano 2014, 1, (4), 338-346.

22. Merrifield, R. C.; Lead, J. R., Preparation and characterization of three-layer, isotopically labelled core-shell nanoparticles; a tool for understanding mechanisms of bioavailability.

NanoImpact 2016, 2, 54-60.

23. Dolan, J. W., Estimating Resolution for Marginally Separated Peaks. Lc Gc Europe 2014, 27, (9), 480-485. 
Figure 1A and B. Particle diameters and concentrations for six nanoparticles Au@Ag-80 nm, Au-80 nm, Ag-80 nm, Au@Ag-60 nm, Au-60 nm and Ag-60 nm as measured by SPICP-MS are shown. All particles suspensions were diluted to 100,000 part. $\mathrm{mL}^{-1}$ based on the data provided by the manufacturer. The 'total diameter' is SP-ICP-MS data collected in this study, accounting for the core-shell geometry. Manufacturer's data is from by transmission electron microscopy, although no data on sample preparation was provided. Figure 1B show the measured particle numbers from SP-ICP-MS

Figure2. Figure $A$ and $B$ show the $A u$ and Ag signal for a NP mixture containing the nominally Au-80 nm, Ag-80 nm and Au@Ag-80 nm particles respectively. Figure $2 \mathrm{C}$ and D show the Au and $\mathrm{Ag}$ signal for a NP mixture containing the nominally $\mathrm{Au}-60 \mathrm{~nm}, \mathrm{Ag}-60$ nm and Au@Ag-60 nm particles. The dotted black curve corresponds to the expected signal from the monometallic particles and the dashed black curve shows the Gaussian curve from the bimetallic (core-shell) particles.

Figure $3 \mathrm{~A}$ and $\mathrm{B}$ : show particle diameters and particle number concentrations for three particle suspensions as measured from SP-ICP-MS. All three suspensions contain Au@Ag-80 nm and Au-80 nm particles with different ratios of particle number concentrations of $1: 4(20,000: 80,000), 1: 1(50,000: 50,000)$ and $4: 1(80,000: 20,000)$ part. $\mathrm{mL}^{-}$ 1. The total particle diameter for the Au@Ag-80nm particle was calculated from the measured Au and Ag signal and is marked with an *. 
Figure 4A and B: show particle diameters and particle number concentrations for two particle suspensions as measured from SP-ICP-MS. The first suspension, $80 \mathrm{~nm}$ mixture, contains Au-80 nm, Ag-80 nm and Au@Ag-80 nm NPs. While the second mixture, 60 nm mixture, contains Au-60 nm, Ag-60 nm and Au@Ag-60 nm NPs. In Figure 4A the diameters of the Au monometallic particles and the Au core from the Au@Ag NPs can be measured accurately but the Ag signals from the monometallic and bimetallic particles are to close to resolve which signal belongs to which particle. However from the data collected all particle numbers can be calculated and can be seen to be close to the expected vales in Figure 4B 

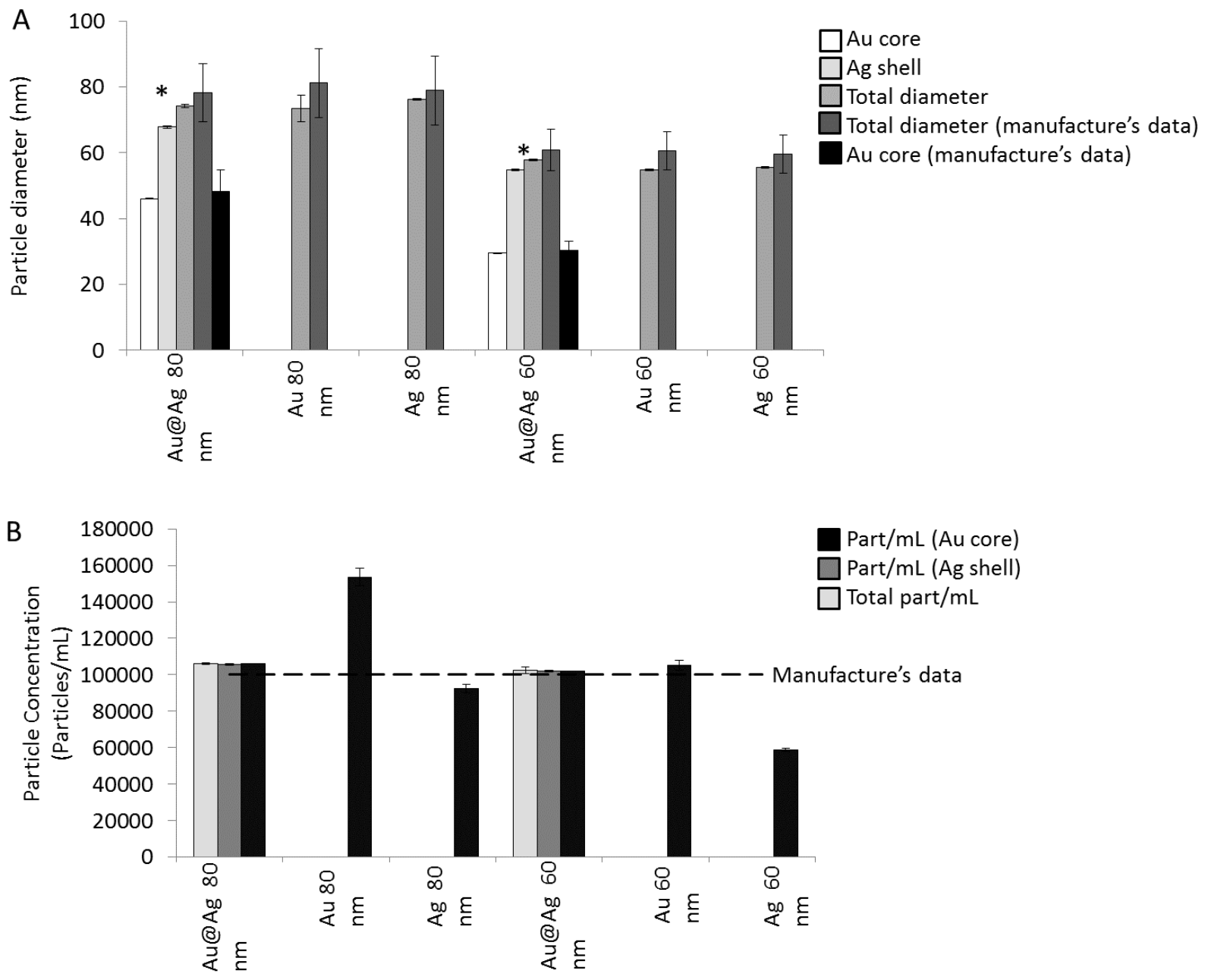
A

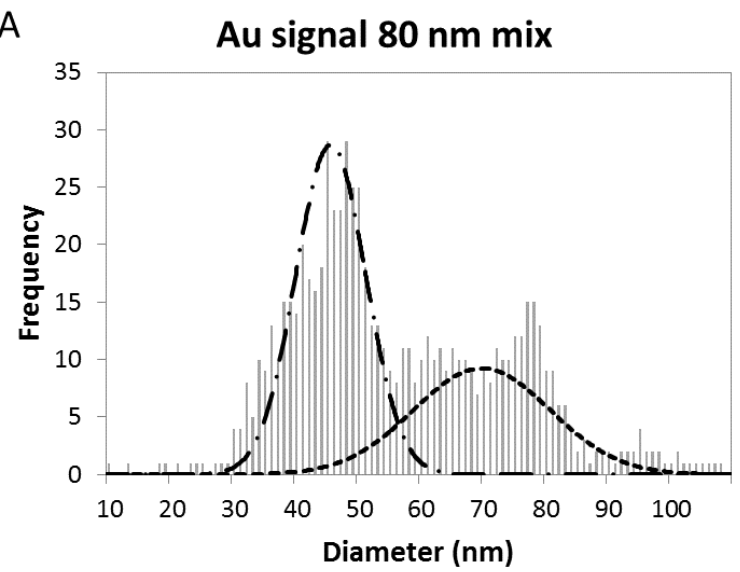

C

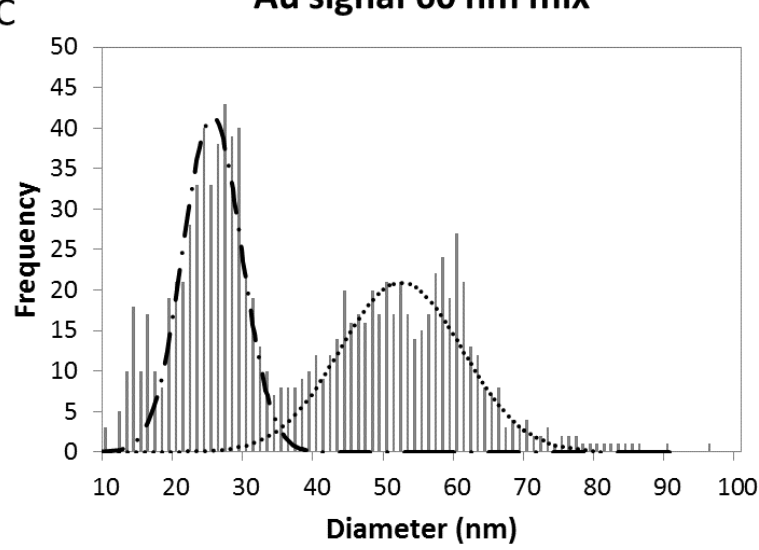

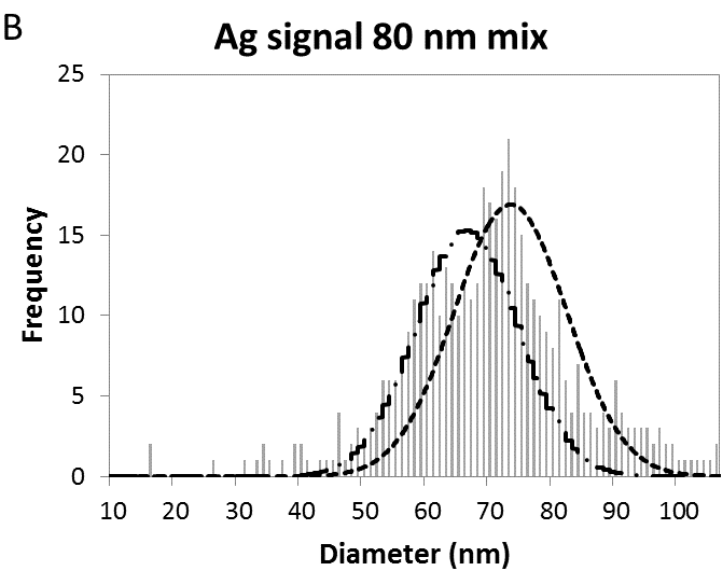

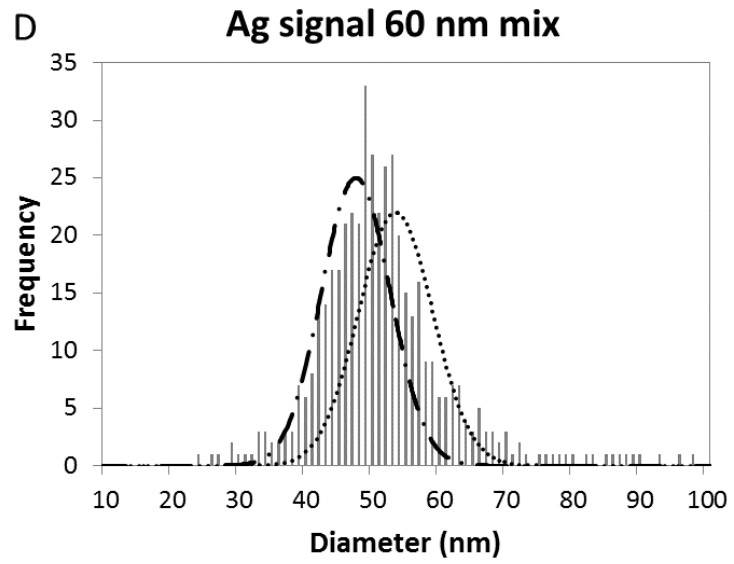



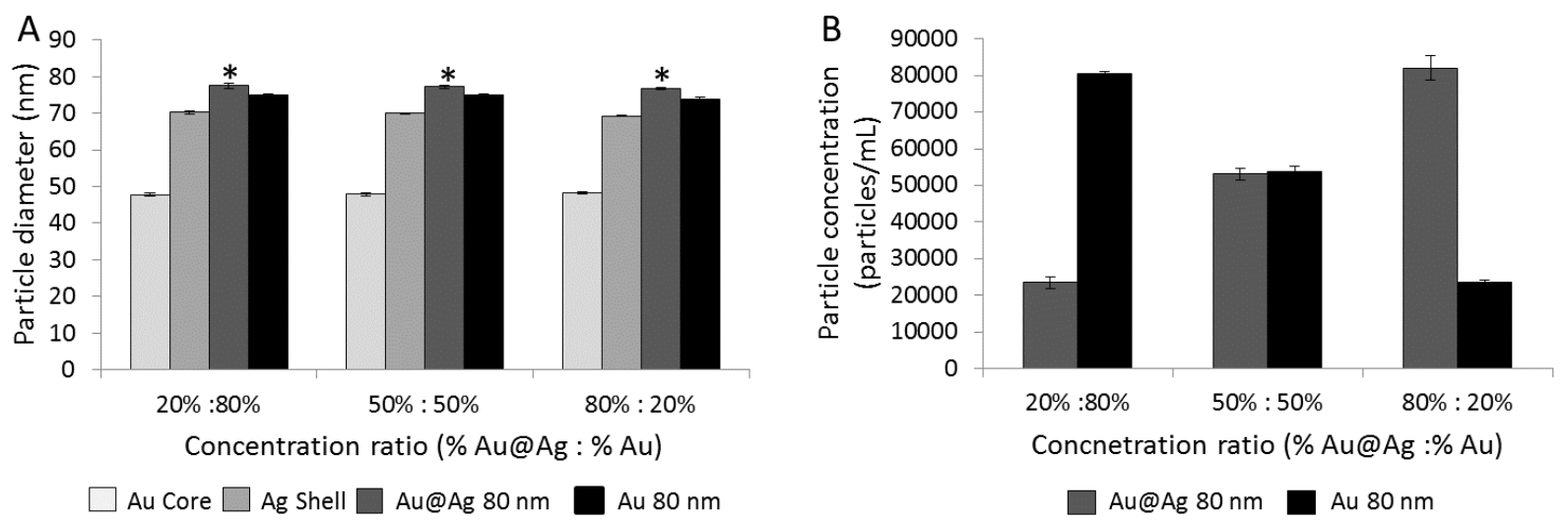

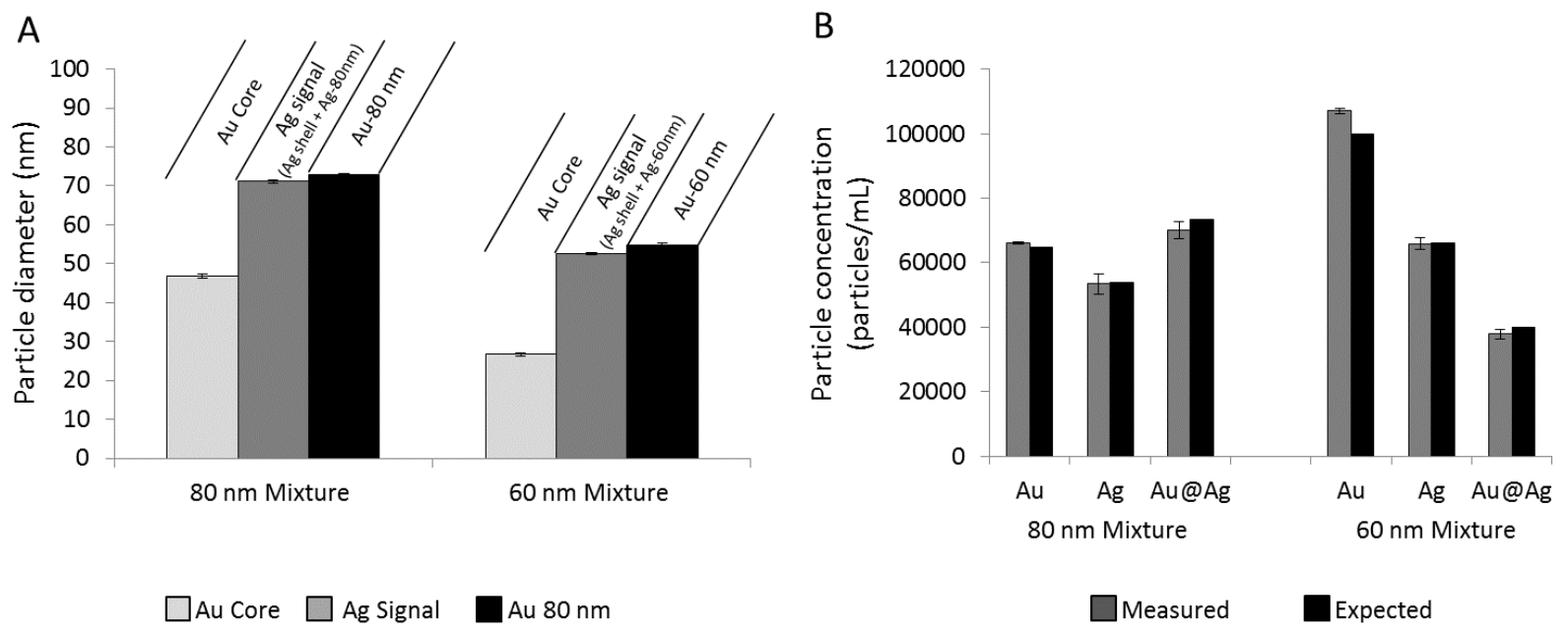


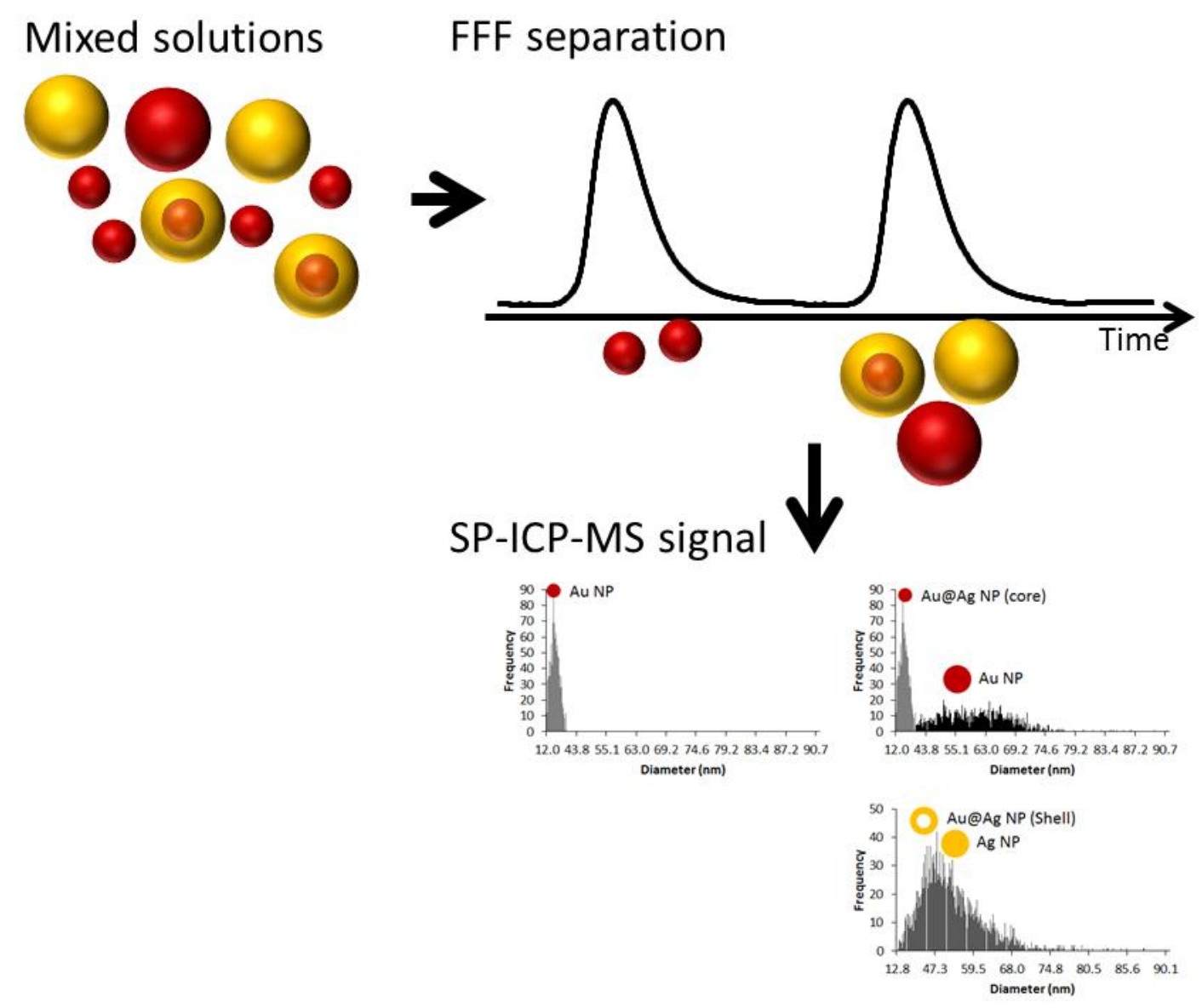

